\title{
Spearman Rank-Order Correlation
}

National Cancer Institute

\section{Source}

National Cancer Institute. Spearman Rank-Order Correlation. NCI Thesaurus. Code C65171.

A distribution-free analog of correlation analysis. Like regression, it can be applied to compare two independent random variables, each at several levels (which may be discrete or continuous). Unlike regression, Spearman's rank correlation works on ranked (relative) data, rather than directly on the data itself. 